\title{
NEUTRON-GAMMA PULSE SHAPE DISCRIMINATION WITH A NE-213 LIQUID SCINTILLATOR BY USING DIGITAL SIGNAL PROCESSING COMBINED WITH SIMILARITY METHOD
}

\author{
Mardiyanto \\ Center for Nuclear Industry Material Technology \\ National Nuclear Energy Agency (BATAN) \\ Puspiptek Area, Serpong, Tangerang 15314 - Indonesia
}

\begin{abstract}
NEUTRON-GAMMA PULSE SHAPE DISCRIMINATION WITH A NE-213 LIQUID SCINTILLATOR BY USING DIGITAL SIGNAL PROCESSING COMBINED WITH SIMILARITY METHOD. Neutron-Gamma Pulse Shape Discrimination with a NE-213 Liquid Scintillator by Using Digital Signal Processing Combined with Similarity Method. Measurement of mixed neutron-gamma radiation is difficult because a neuclear detector is usually sensitive to both radiations. A new attempt of neutron-gamma pulse shape discrimination for a NE-213 liquid scintillator is presented by using digital signal processing combined with an off-line similarity method. The output pulse shapes are digitized with a high speed digital oscilloscope. The $n-\gamma$ discrimination is done by calculating the index of each pulse shape, which is determined by the similarity method, and then fusing it with its corresponding pulse height. Preliminary results demonstrate good separation of neutron and gamma-ray signals from a NE-213 scintillator with a simple digital system. The results were better than those with a conventional rise time method. Figure of Merit is used to determine the quality of discrimination. The figure of merit of the discrimination using digital signal processing combined with of line similarity method are $1.9 ; 1.7 ; 1.1 ; 1.1$; and 0.8 ; on the other hand by using conventional method the rise time are $0.9 ; 0.9 ; 0.9 ; 0.7$; and 0.4 for the equivalent electron energy of $800 ; 278 ; 139 ; 69$; and $30 \mathrm{keV}$.
\end{abstract}

Keywords : Neutron-gamma discrimination, Similarity method, Digital signal processing, Liquid scintillator, Rise time method

\section{INTRODUCTION}

Although liquid scintillators are very poor gamma-ray detectors due to their low atomic number $Z$, they have some advantages for specific applications, such as low energy beta emitter of ${ }^{14} \mathrm{C}$ measurement[1], alpha emission of ${ }^{222} \mathrm{Rd}$ measurement[2], etc.

Besides those mentioned above reasons, liquid scintillators become popular because they are naturally flexible media. Thus, they can be applied in a big size for measuring high neutron energy[3]. On the other hand, they can be filled into a capillary detector for reconstructing of ionizing particle tracks[4]. Moreover, the liquid scintillators are relatively cheap detectors.

In contrast to the advantages of the use of liquid scintillators, they are usually hazardous. In order to minimize the hazardous, recently a research work to look for less hazard materials has been conducted[5]. 
One of the intensive use of liquid scintillators is for the neutron detection. The low atomic number makes these detectors effective for detecting neutrons. The main problem of using these detectors for neutron detection is that the detectors also sensitive to gamma-ray background. Many research works have been conducted to reject the gamma-ray background under neutron detection[6-9].

In this paper, we propose a new method based on a similarity with a fast digital for neutron-gamma ray discrimination especially for the lower part of the neutron energy which is usually difficult to discriminate using conventional methods.

\section{THEORY}

\section{Similarity}

In digital image processing, one of the similarity methods can be approached from a detail indication based on the structural features such as edges, skeleton, medial axis, convex hull, object symmetry etc. In our approach here, we use the similarity to describe how close the patterns of two different pulse shapes are when each of the pulses is represented by a vector. The present similarity $\mathrm{S}(\mathrm{X}, \mathrm{Y})$ is defined as follows $[10]$;

$$
S(X, Y)=\frac{(X, Y)}{|X||Y|}=\cos \theta
$$

where $\mathrm{X}$ is a pattern vector to be identified and $\mathrm{Y}$ is an appropriate reference or discriminant vector, $(\mathrm{X}, \mathrm{Y})$ is the scalar product, $|\mathrm{X}|$ and $|\mathrm{Y}|$ are the norms of the vectors, and $\theta$ is the angle between the two vectors. Here, the angle $\theta$ is used as an index of the pulse shape instead of the cosine.

In the digital system, a pulse shape can be represented by a vector $\mathrm{X}=\left(\mathrm{x}_{1}, \mathrm{x}_{2}, \mathrm{x}_{3}, \ldots \ldots \ldots \mathrm{x}_{\mathrm{n}}\right)$, where $\mathrm{n}$ is the number of the elements of the vector $\mathrm{X}$ (Figure 1). The elements of $\mathrm{X}$ can be obtained directly from the data measured by a digitizer. 


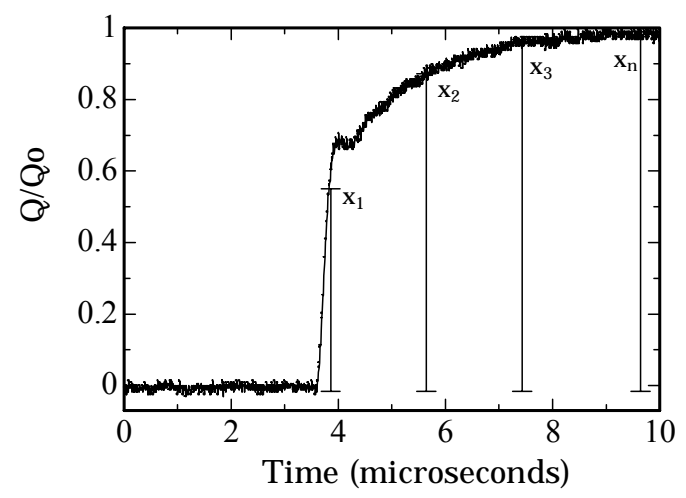

Figure 1. Vector elements from abscissa.

\section{Liquid scintillator pulse shape analysis}

Many published papers concerning the liquid scintillators investigate the discussion of various processes which lead to light emission or which prevent it[5-7]. There is other source of information which is useful especially for a charged particle identification namely the behavior of light emission based on its slow decay time as the result of a single fast particle interaction.

The energy transfer process from the fast particle to the molecules of the scintillator happens in a very short time, that is in the order of $10^{-10} \mathrm{sec}$. The profile of the light pulse shape is of the form[11] :

$$
I(t)=I_{0}\left(e^{-t / \tau}-e^{-t / \tau_{1}}\right)
$$

where : $\tau_{1}=$ the rise time (nsec.),

$\tau=$ the decay time (nsec.).

and by using eq.(2) the plot of the pulse shape I(t) can be made, as shown in Figure 2.

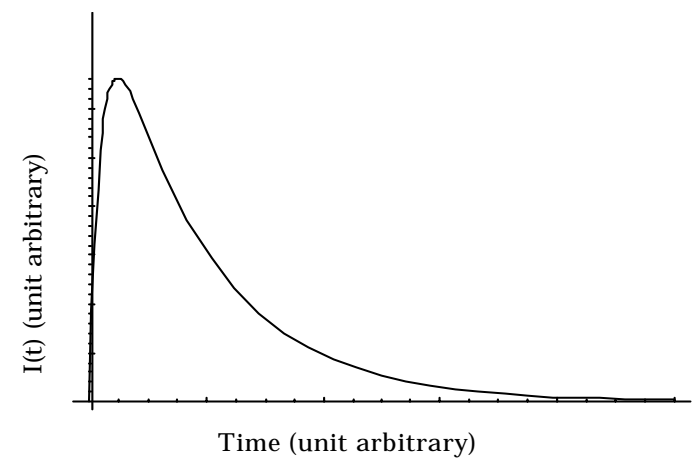

Figure 2. Scintillator output pulse shape. 
Here, the rise time depends upon the solute concentration and decreases with increasing concentration. On the other hand, for the decay time, there will be two components namely fast decay and slow decay due to the fluorescence and phosphorescence process, respectively. In order to make clearer about the excitation/de-excitation process, it can be shown schematically in Figure 3. In this case, the slow decay is strongly influenced by the $\mathrm{dE} / \mathrm{dx}$ of the radiation. Based on this phenomena, eq. (2) can be restated as :

$$
I(t)=A\left(e^{-t / \tau_{f}}-e^{-t / \tau_{1}}\right)+B\left(e^{-t / \tau_{s}}-e^{-t / \tau_{1}}\right)
$$

where $: \tau_{f}, \tau_{\mathrm{s}}, \mathrm{A}$, and $\mathrm{B}$ are the fast decay time constant, slow decay time constant,

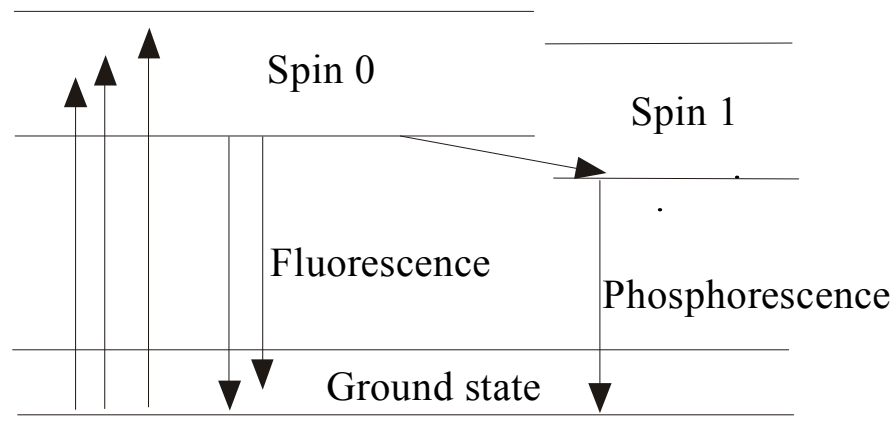

Figure 3. The excitation/de-excitation process.

fluorescence fraction, and phosphorescence fraction, respectively. The plot of $\mathrm{I}(\mathrm{t})$ is shown in Figure 4.

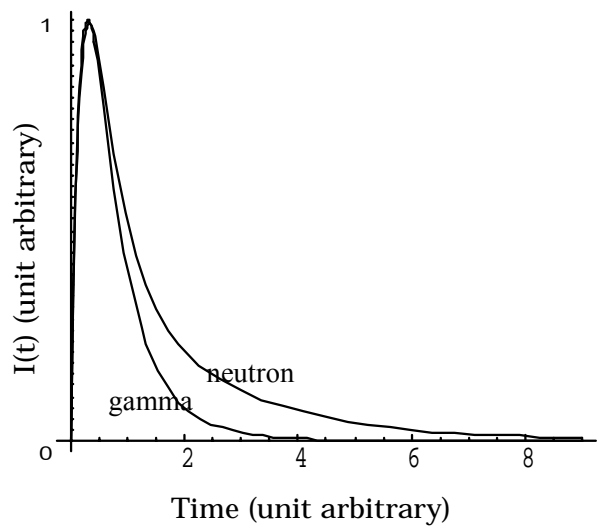

Figure 4. Scintillator output pulse shapes for two charged particles with different $\mathrm{dE} / \mathrm{dx}[6-9]$. 
Because the slow decay produces a small fraction of light, one can, therefore, make use this dependence to separate different kinds of particles which deposit the same energy in the detector medium with the different $\mathrm{dE} / \mathrm{dx}$. Many papers, which use this phenomenon for particle identification have been published[6-9].

\section{EXPERIMENTAL METHOD}

In this experiment, ${ }^{252} \mathrm{Cf}$ neutron source was placed $10 \mathrm{~cm}$ in front of a liquid scintillation detector. The detector used was a NE213 liquid scintillator which was coupled with a photomultiplier (Hamamatsu H6614-01 type). The bias voltage of 1200 volts for the photomultiplier was supplied by a high voltage source C.A.E.N Mod.471.

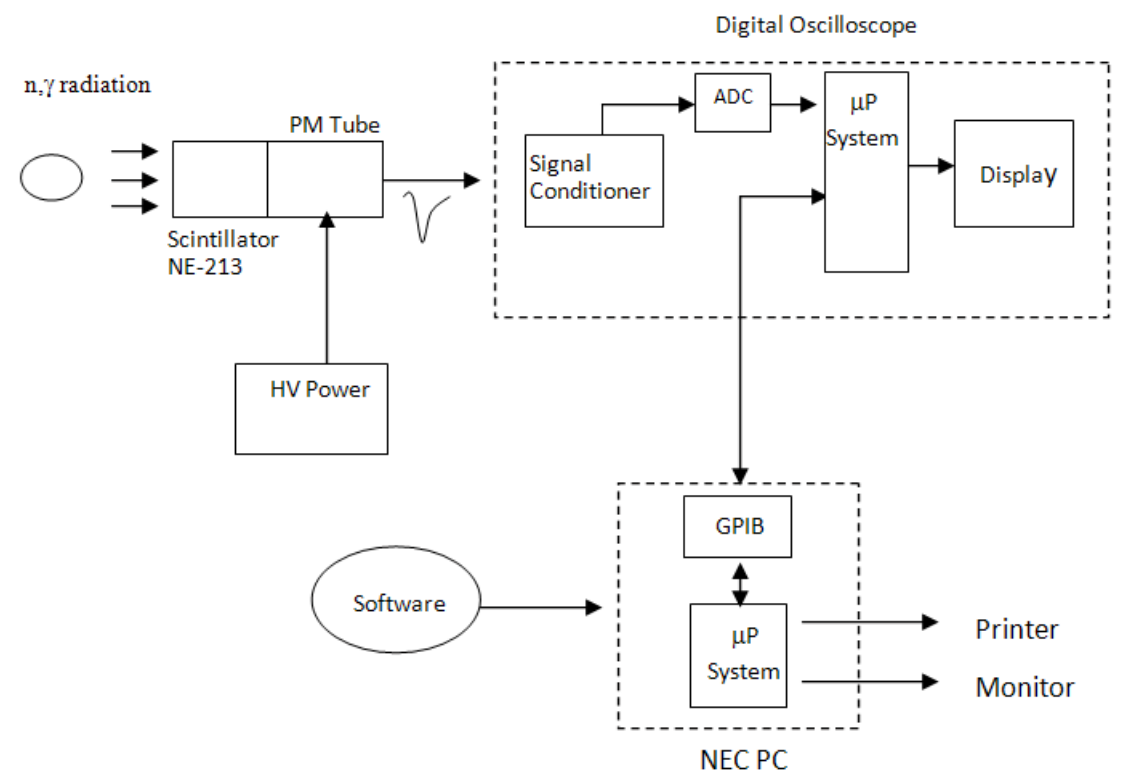

Figure 5. Schematic drawing of the measurement system.

The output of the photomultiplier was connected directly to a digital oscilloscope (Tektronix TDS 782A) where each of the pulse shape was digitized with the sampling frequency of $2 \mathrm{Gsampling} / \mathrm{sec}$.

The sampled data were saved in a NEC PC through a GPIB. In order to minimize the use of the PC memory, only the pulse shape of the digitized data, namely 181 points, were saved. A workstation was then used for analyzing the pulse shape. The whole system is shown in Figure 5. 


\section{RESULTS AND DISCUSSION}

\section{Experiment With Medium Size NE-213 (Dia. :5.08 cm and Length: $5.08 \mathrm{~cm})$}

Based on eq.(3), when a neutron and $\mathrm{X} / \gamma$-rays enter a liquid scintillator, the output pulse shape will have different shapes especially in their decay parts. Figure 6 shows two kinds of pulse shape that are expected to be the pulse shapes of the neutron and the photon. Although there is an interference noise, we can determine the two pulse shapes.

In the digital system, a pulse shape can be represented by a vector $\mathrm{X}=\left(\mathrm{x}_{1}, \mathrm{x}_{2}, \mathrm{x}_{3}, \ldots \ldots \ldots \mathrm{x}_{\mathrm{n}}\right)$, where $\mathrm{n}$ is the number of the elements of the vector $\mathrm{X}$. The elements of $\mathrm{X}$ can be obtained directly from the data measured by a digitizer.

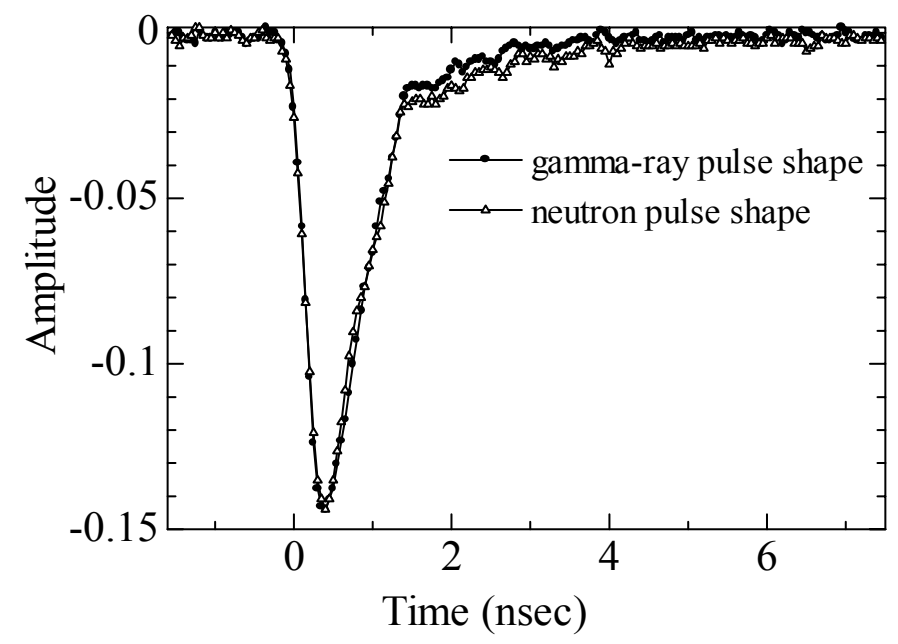

Figure 6. Gamma-ray pulse shape and neutron pulse shape.

In these experiments, a detector with the diameter and length of $5.08 \mathrm{~cm}$ and $5.08 \mathrm{~cm}$ was used. In order to know the dynamic range of the discrimination capability, the measurements were done in several volt/div ranges. With the system arrangement shown in Figure 5, some measurements were done with the different volt/div. ranges i.e. $50 \mathrm{mV} / \mathrm{div}, 20 \mathrm{mV} / \mathrm{div}$., and $10 \mathrm{mV} / \mathrm{div}$.

By using eq.(1) and taking a pulse shape from the measured data as the discriminant vector, the $\theta$ value of each pulse shape of both gamma-rays and neutrons was calculated. As the discriminant vector, it can be chosen either from the gamma-ray pulse shape or neutron pulse shape. Here, neutron pulse shape was used as the driscriminant vector. The elements of the pattern vector $\mathrm{X}$ and the discriminant vector $\mathrm{Y}$ were chosen from the sampled data 
namely from the point number 69 to 181 . Point number 69 was chosen as the starting point for the extracted feature of each pulse shape because the two kinds of pulse shapes (gamma-ray and neutron) started to deviate from the point. The results of the calculation are shown in Figures. 9(a) (b), and (c).

In order to know the electron energy equivalent produced by recoil proton, it is necessary to make an electron equivalent energy calibration. For this purpose, the Compton edge of ${ }^{137} \mathrm{Cs}$ was used. The measurement was done by setting the digital oscilloscope with the same Volt/div scale. The Compton edge is shown in Figure 7. The energy of the Compton edge can be calculated as :

$$
h v-E_{c}=662-\frac{h v}{1+\frac{2 h v}{m_{o} c^{2}}}
$$

where : hv $=$ the energy of the photon,

$\mathrm{E}_{\mathrm{c}}=$ the energy of the back scattered photon, $\mathrm{m}_{0} \mathrm{c}^{2}=$ the rest-mass energy of the electron.

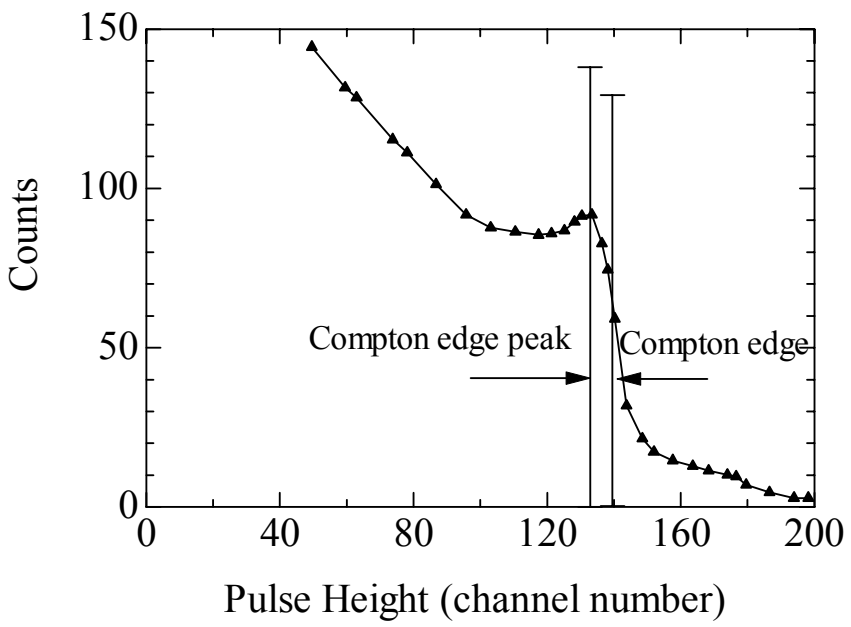

Figure 7. Compton edge of ${ }^{137} \mathrm{Cs}$.

There are two simple ways of determining the Compton edge. First, Compton edge channel is determined by using $\mathrm{Nc}=\mathrm{Np}+50(\mathrm{FWHM} / \mathrm{Np})[12]$, where $\mathrm{Nc}$ is the channel number of the Compton edge, $\mathrm{Np}$ is the channel number of the edge peak, and the FWHM is the full wave at half maximum of the Compton edge. Second, Compton edge is determined by using $\mathrm{Nc}=\mathrm{Np}+1.177 \sigma[13]$, where $\mathrm{Nc}, \mathrm{Np}$ are the same as the definition mentioned 
above while $\sigma$ is standard deviation of the edge peak. Those two ways gave almost the same results namely the different was less then one channel.

Thus, by using eq.(4) and one of the two above ways, the energy of the Compton edge of ${ }^{137} \mathrm{Cs}$ is $477 \mathrm{keV}$ corresponding to 137 channel.

A quantity that is used for representing the discrimination power is called as a figure of merit (FOM). The definition of figure of merit is given as :

$$
F O M=\frac{T_{n-\gamma}}{W_{n}+W_{\gamma}}
$$

where : $\mathrm{T}_{\mathrm{n}-\gamma}, \mathrm{W}_{\gamma}$, and $\mathrm{W}_{\mathrm{n}}$ are the distance between the gamma-ray and neutron peak, the FWHM of gamma-rays, and the FWHM of neutrons (Figure 8).

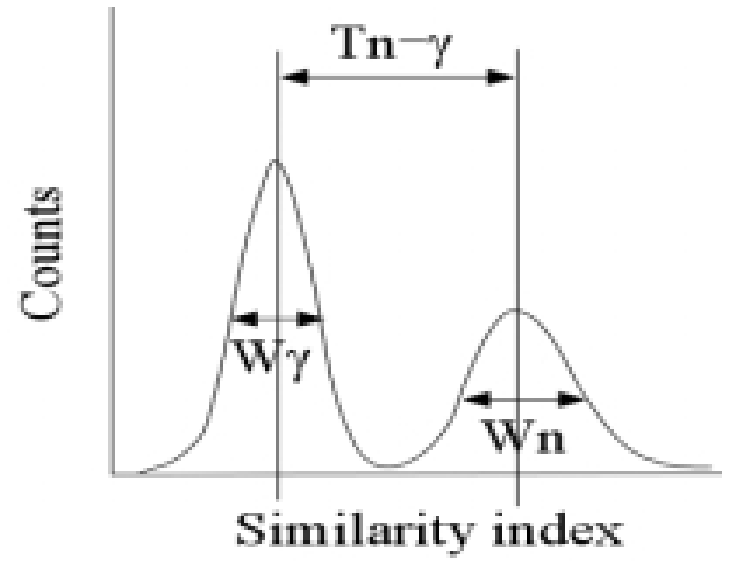

Figure 8. A sketch for determining figure of merit (FOM). 


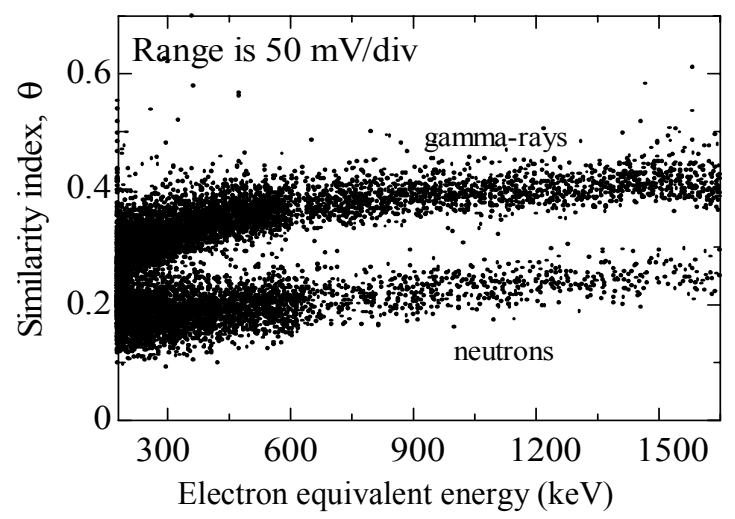

(a)

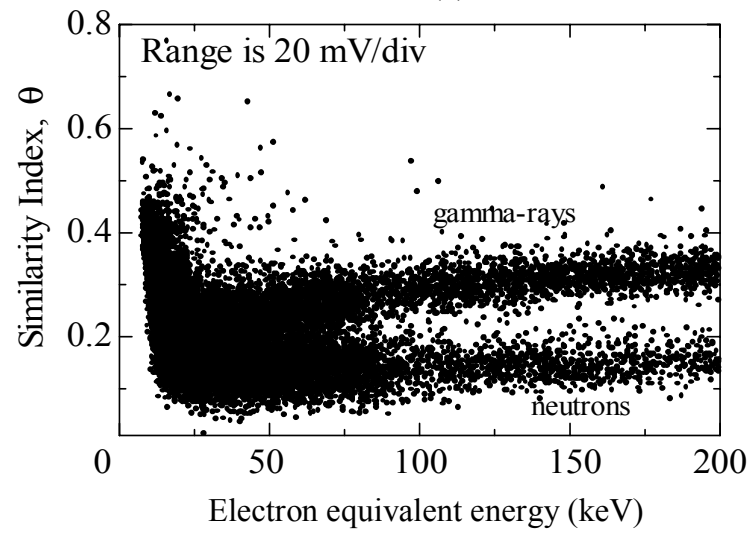

(b)

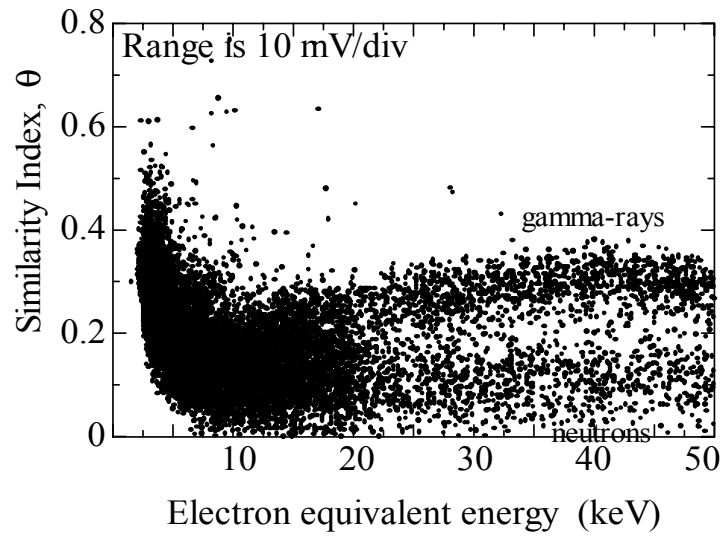

(c)

Figure 9. The results of discrimination for three different volt/div ranges. 
Based on the calculations using eq.(4) and the result of electron equivalent energy calibration (Figure 9), the figure of merits of the discrimination were determined for several neutron energy values. The results are illustrated in Figure 10.

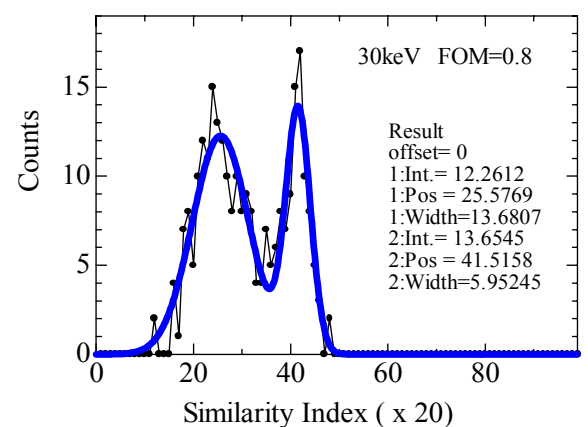

(a)

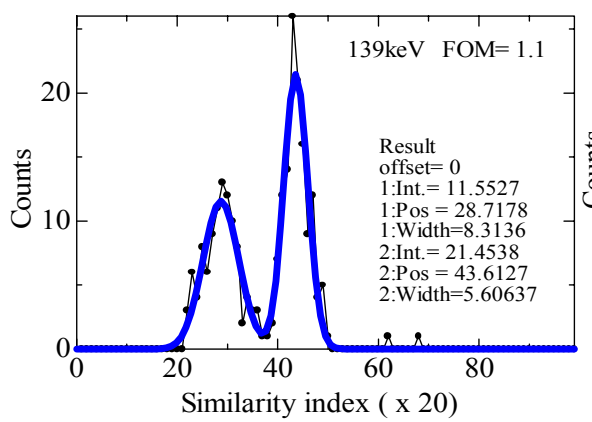

(c)

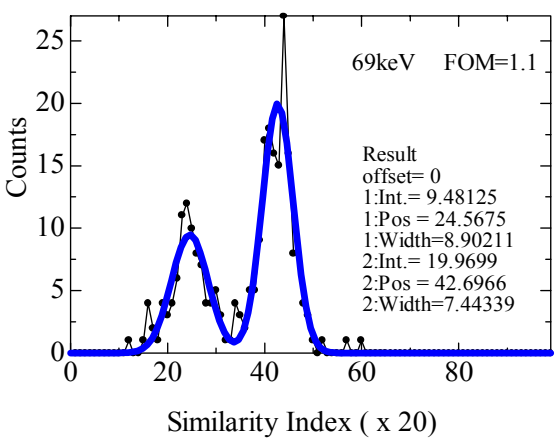

(b)

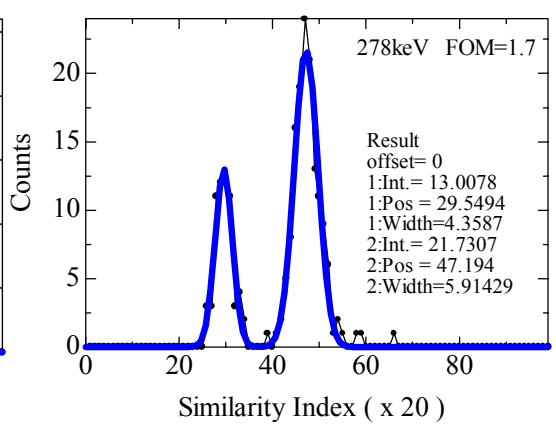

(d)

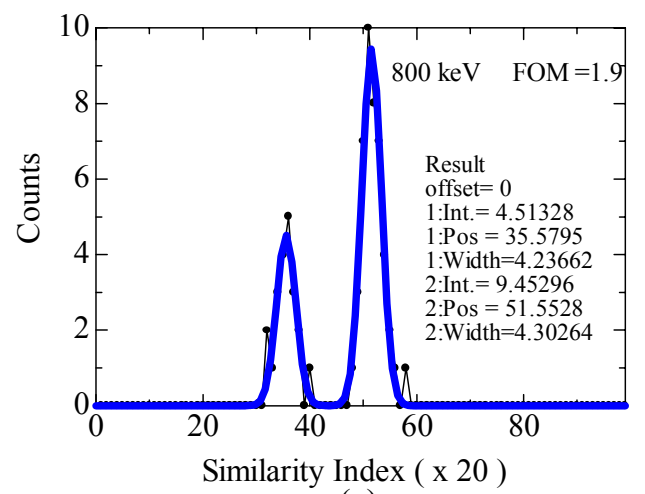

(e)

Figure 10. Figure of merit for different neutron energies. 
Neutron-gamma discrimination can be done easily when their energy is high $(>3 \mathrm{MeV})[14]$. This work becomes more difficult with the decreasing energy. From the results shown in Figure 10, the digital method that was combined with the similarity method could make discrimination of mixed neutron-gamma energy spectra as low as electron equivalent energy of $30 \mathrm{keV}$ with the figure of merit of 0.8 .

\section{Neutron-gamma discrimination with rise time method}

In order to make comparison of the discrimination results of the present method with a conventional one, the neutron-gamma discrimination with a rise time method was also done. The results of the neutron-gamma discrimination with the rise time method using the cylindrical NE-213 (diameter : $5.08 \mathrm{~cm}$, length : $5.08 \mathrm{~cm}$ ) is shown in Figure 11. Here, for the energy calibration a ${ }^{137} \mathrm{Cs}$ gamma-ray source was also used.

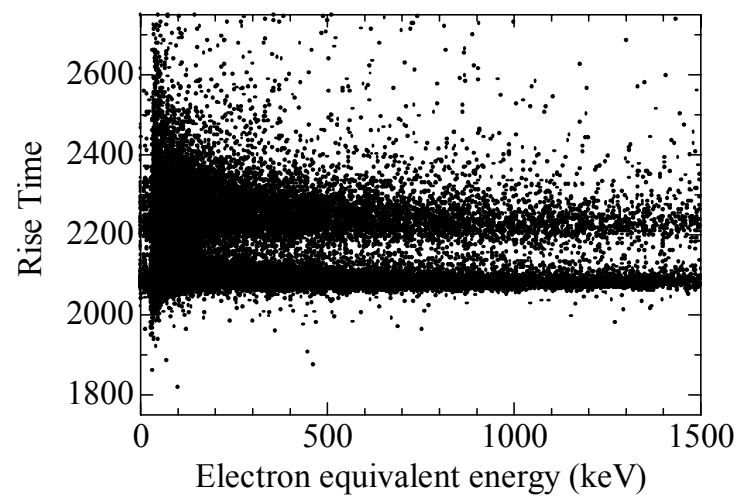

Figure 11. Neutron-gamma discrimination results using rise time method.

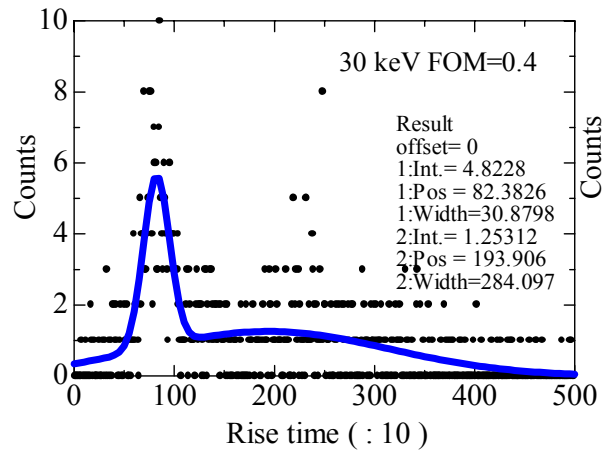

(a)

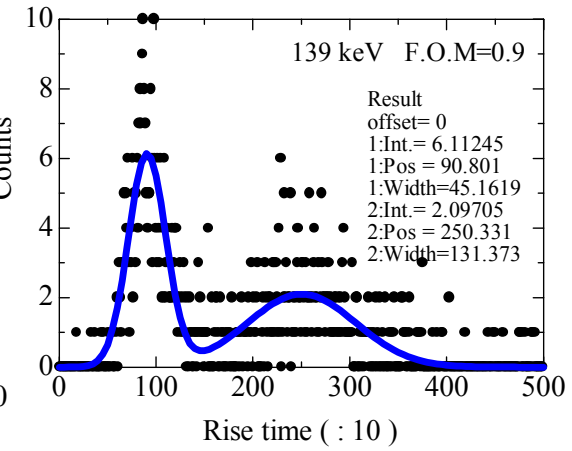

(b)

Figure 12. Figure of merits, electron equivalent energy of (a) $30 \mathrm{keV}$ and (b) $139 \mathrm{keV}$. 
Figure of merits for the electron equivalent energy of $30 \mathrm{keV}, 69 \mathrm{keV}$, $139 \mathrm{keV}, 278 \mathrm{keV}$, and $800 \mathrm{keV}$ were calculated. Figures 12 . (a) and (b) show the figure of merits for the electron equivalent energy of $30 \mathrm{keV}$ and $139 \mathrm{keV}$.

Table 1. FOM comparison between digital combined with similarity and rise time method

\begin{tabular}{|lc|c|c|c|c|c|}
\hline \multirow{2}{*}{ Method } & \multicolumn{5}{|c|}{ Electron equivalent energy (keV) } \\
\cline { 3 - 7 } & 800 & 278 & 139 & 69 & 30 \\
\hline $\begin{array}{l}\text { Digital combined with } \\
\text { similarity }\end{array}$ & 1.9 & 1.7 & 1.1 & 1.1 & 0.8 \\
\hline Rise time method & 0.9 & 0.9 & 0.9 & 0.7 & 0.4 \\
\hline
\end{tabular}

The results of the comparison between the present method with the rise time method for all above mentioned energies are shown in Table 1. From Table 1, it is shown that the present method gave better figure of merits.

\section{CONCLUSION}

A simple method for neutron-gamma-ray pulse shape discrimination has been proposed by using a similarity method. Through the energy calibration and the figure of merit evaluation, it has been confirmed that this method resulted in very good neutron-gamma discrimination even for the lower energy part, and the $\mathrm{FOM}=0.8$ was obtained at the electron equivalent energy of $30 \mathrm{keV}$.

Compared to the rise time method (conventional method), the present method gave better figure of merits over all of the range of the electron equivalent energies under concern.

Finally, the present off-line method, in particular the similarity index, is very simple and effective to characterize the pulse shape, so that it may be possible to be used in the real time method.

\section{REFERENCES}

1. ALIMONTI, G., et al., Physics Letters, B 422, 349 (1998).

2. TOME, F.V., LOZANO, J.C., ESCOBAR, V.G., Nucl. Instr. and Meth. A 425,.589 (1999).

3. ITO, S., et al., Nucl. Instr. and Meth. A 354,.475 (1995).

4. BAY, A., MARTEllOtTI, G., Nucl. Instr. and Meth., A 384, 179 (1996). 
5. MAJEWSKI, S., et al., Nucl. Instr. and Meth. A 414, 289 (1998).

6. LEE, J.H., LEE, C.S., Nucl. Instr. and Meth., A 402, 147 (1998).

7. WOLSKI, D., et al., Nucl. Instr. and Meth. A 360, 584 (1995).

8. ZECHER, P.D., et al., Nucl. Instr. and Meth., A 401, 329 (1997).

9. JORDANOV, V.T., KNOLL, G.F., IEEE Trans. on Nucl. Sci., 42 (4), 683 (1995).

10. SAKAI, H., et al., Nucl. Instr. and Meth., A 421, 316 (1999).

11. KALlmaNN, H., FURST, M., "Proceedings of Liquid Scintillation Counting", 3 (1957).

12. HRISTOVA, A.V., et al., Applied Radiation and Isotopes, 41 (9), 887 (1990).

13. CHIKKUR, G.C., UMAKANTHA, N., Nucl. Instr. and Meth., 107, 201 (1973).

14. DIETZE, G., KLEIN, H., Nucl. Instr. and Meth., 193, 549 (1982). 